\title{
Tratamento aditivo do ceratocone por 'crosslinking' do colágeno após implante de anel de Ferrara
}

\section{Addictive treatment of keratoconus with collagen crosslinking after Ferrara ring implant}

Edna Almodin ${ }^{1}$, Steve Arschinoff ${ }^{2}$, Juliana Almodinn³, Paulo Ferrara ${ }^{4}$

\section{Resumo}

Objetivo: Este trabalho tem como objetivo relatar experiência com utilização do crosslinking após implante de anel intracorneano de Ferrara (Ferrara Ophtalmic) visando a melhora refrativa e estabilização corneana. Métodos: Seis pacientes com idade entre 26 e 38 anos foram submetidos ao tratamento com crosslinking após implante de anel de Ferrara. Cinco pacientes receberam 2 segmentos de anel e um paciente recebeu um segmento. Depois de 4 a 55 meses os pacientes foram submetidos a aplicação do "crosslinking" com ultra-violeta de acordo com o protocolo estabelecido por Seiler \& cols. Resultados: Quatro olhos apresentaram alteração progressiva da refração e 2 apresentaram refração sem alteração progressiva. A topografia mostrou queda acentuada da curvatura e o estudo da lâmpada de fenda mostrou anel em boa profundidade durante um ano de pós-operatório em $100 \%$ dos olhos. A microscopia especular não mostrou alteração significativa e a acuidade visual se mostrou semelhante ao pré-operatório. Conclusão: O crosslinking poderá ajudar a manter ceratocones estáveis, não-somente nos casos de progressão, mas também nos cones evolutivos com implante de anel.

Descritores: Ceratocone/cirurgia; Anel intraestromal; Córnea/cirurgia; Córnea/ patologia; Acuidade visual; Agentes fotossensibilizantes/uso terapêutico; Riboflavina/ uso terapêutico; Raios ultravioleta; Topografia da córnea

\footnotetext{
'Mestre, Diretora do Hospital de Olhos Provisão - Maringá (PR), Brasil;

2 Professor da Universidade de Toronto-Canadá York Finch General Hospital, Toronto;

${ }^{3}$ Médica oftalmologista do Hospital de Olhos Provisão - Maringá (PR), Brasil; Assistente do Departamento de Glaucoma do Instituto de Oftalmologia Tadeu Cvintal - São Paulo (SP), Brasil;

${ }^{4}$ Professor da Universidade Federal de Minas Gerais - UFMG - Belo Horizonte (MG), Brasil;
} 


\section{INTRODUÇÃO}

D esde a década de 40, vários pesquisadores têm se esforçado na tentativa de estabilizar asectasias de córneas evitando o transplante. Recentemente bons resultados têm sido obtidos com implante de anel intraestromal ${ }^{(1)}$. O implante de anel intra-estromal tem demonstrado estabilização corneana por longo período e tem ajudado a diminuir de maneira significativa as filas de pacientes em espera para transplante ${ }^{(2)}$. Algumas formas de anel intraestromal têm sido relatadas ${ }^{(3)}$, porém sempre com o intuído de evitar o transplante, principalmente nos pacientes com intolerância à lentes de contato $^{(4)}$. Apesar dos resultados animadores nas últimas décadas, alguns autores em longo follow-up têm relatado em $5 \%$ dos casos piora na acuidade visual (AV) se comparado ao préoperatório $^{(5)}$. Tem sido demonstrado a possibilidade de enrijecimento da córnea através do aumento do número de ligações cruzadas entre as fibras de colágeno, com a utilização de irradiação ultravioleta (UV) associada ao uso da substância fotossensibilizadora, riboflavina (crosslinking) ${ }^{(6)}$. Relatos mais recentes têm demonstrado que esta associação provoca apoptose dos queratócitos atingindo até 300 micras de profundidade na córnea. Esta população de queratócitos é recomposta em até 6 meses, e desde que se respeite o limite de 400 micras na espessura corneana não foi evidenciado danos ao endotélio, cristalino ou retina ${ }^{(7)}$. Pesquisadores têm demonstrado a utilização do crosslinking em keratectasias após Lasik ${ }^{(8)}$. O recente alargamento das indicações na utilização desta nova metodologia tem sido utilizado no tratamento de úlceras corneanas, melting e ceratopatia bolhosa ${ }^{(9)}$.

Este trabalho tem o propósito de relatar nossa experiência com utilização do crosslinking após implante de anel intracorneano de Ferrara (Ferrara Ophtalmic) visando melhora refrativa e estabilização corneana neste grupo de pacientes.

\section{Métodos}

Após aprovação pelo comitê de ética do Hospital Almodin Ltda. e consentimento esclarecido informado, seis pacientes com idade entre 26 e 38 de idade, sendo 5 masculinos e 1 feminino foram submetidos ao tratamento com crosslinking após implante de anel de Ferrara. Dentre estes, quatro (67\%) tiveram piora da AV e aumento progressivo na refração após três meses do implante de anel, e dois $(33 \%)$ pacientes tiveram extrusão do segmento inferior após 2.2 e 2.8 anos após o implante e apesar de ainda não apresentarem piora progressiva foram incluídos no grupo de estudo.

O implante de anel de Ferrara foi realizado com anestesia local (colírio anestésico). A incisão foi realizada no eixo de maior curvatura corneana na profundidade de $80 \%$ da paquimetria local. Foi realizado tunelização na zona óptica de $5 \mathrm{~mm}$. Cinco destes pacientes receberam 2 segmentos de anel e um paciente recebeu apenas 1 segmento inferior. No pós-operatório foi utilizado anti-inflamatório não hormonal (cetrolaco de trometamina) de 15/ 15 minutos por $3 \mathrm{hs}$, associação de corticóide com antibiótico (prednisona e gatifloxacino) por 7 dias e anti-inflamatório não hormonal cetrolaco de trometamina de $15 / 15$ minutos por $3 \mathrm{~h}$. Entre 4 a 55 meses após o implante de Anel, os pacientes foram submetidos à aplicação do crosslinking com UVA e Riboflavina, de acordo com protocolo estabelecido por Seiler \& cols. ${ }^{(6)}$. Suscintamente, após a desepitelização corneana da zona óptica central de $9 \mathrm{~mm}$, a riboflavina foi aplicada de $5 / 5$ minutos durante 30 minutos e intercalada com lavagem de solução balanceada de BSS a cada 2.5 minutos após cada aplicação de riboflavina.

Após 30 minutos, os pacientes eram examinados na lâmpada de fenda para reconhecimento de flare amarelado na câmara anterior e depois expostos ao UVA (370nm, 3nW / $\mathrm{cm}^{2}$ ) por mais 30 minutos acrescidos da mesma dose de riboflavina, uma gota de $5 / 5$ minutos ( 6 aplicações) intercaladas por lavagem com solução balanceada de BSS após 2.5 minutos de cada aplicação da riboflavina. Após o término do procedimento utilizamos lentes de contacto gelatinosas terapêutica por 5 a 7 dias no pósoperatório até reepitelização total da córnea. Os pacientes fizeram uso de colírios de antibiótico por uma semana e lágrimas artificiais por um mês, associado analgésico oral por $24 \mathrm{~h}$. Utilizamos corticóide local (prednisona) somente após a reepitelização total da córnea por mais uma semana.

O seguimento pós-operatório dos pacientes foi realizado no primeiro, terceiro, sexto e décimo 
Tabela 1

Acuidade visual média e desvio padrão

\begin{tabular}{lccccc}
\hline & Pré op. & $\mathbf{1}$ mês & $\mathbf{3}$ meses & $\mathbf{6}$ meses & $\mathbf{1 2}$ meses \\
\hline Média & 0,073 & 0,532 & 0,53 & 0,46 & 0,52 \\
DP & 0,036 & 0,223 & 0,116 & 0,202 & 0,117
\end{tabular}

Tabela 3

\section{Progressão da curvatura máxima pelo Pentacan}

\begin{tabular}{lc}
\hline & Pentacan \\
Pré-operatório & 49,4 \\
1 mês & 39,8 \\
3 meses & 41,3 \\
6 meses & 42,2 \\
1 ano & 42,9 \\
\hline
\end{tabular}

Tabela 5

Refração média e desvio padrão

\begin{tabular}{lccc}
\hline & Pré-operatório & $\mathbf{1}$ mês & $\mathbf{1 2}$ meses \\
\hline Média & $-4,41$ & $-4,17$ & $-3,38$ \\
Desvio padrão & 4,98 & 5,01 & 4,47 \\
\hline
\end{tabular}

Tabela 2

Topografia (Pentacam) pré e pós-anel de Ferrara

\begin{tabular}{lccccc}
\hline & Pré-op. & $\mathbf{1}$ mês & $\mathbf{3}$ meses & $\mathbf{6}$ meses & $\mathbf{1}$ ano \\
\hline Média & 52,52 & 46,30 & 46,50 & 46,40 & 46,02 \\
Desvio Padrão & 6,67 & 4,88 & 4,32 & 3,04 & 3,10 \\
\hline
\end{tabular}

Tabela 4

Acuidade visual pré- e pós-operatório em 'Crosslinking'

\begin{tabular}{lccc}
\hline & $\begin{array}{c}\text { Pré- } \\
\text { operatório }\end{array}$ & $\begin{array}{c}\text { 1 mês } \\
\text { pós op. }\end{array}$ & $\begin{array}{c}\text { 12 meses } \\
\text { pós op. }\end{array}$ \\
\hline Média & 30,83 & 30,83 & 23,33 \\
Desvio padrão & 14,97 & 12,01 & 8,16 \\
\hline
\end{tabular}

Tabela 6

Media topográfica pré e pós-operatória

\begin{tabular}{lccc}
\hline & Pré-operatório & 1 mês & 12 meses \\
\hline Média & 47,45 & 47,43 & 47,1 \\
Desvio padrão & 4,44 & 3,95 & 3,56 \\
\hline
\end{tabular}

Tabela 7

\begin{tabular}{lccc}
\multicolumn{4}{c}{ Microscopia especular } \\
\hline & Pré-operatório & 1 mês & 12 meses \\
\hline Média & 2857 & 2886 & 2877 \\
Desvio padrão & 298 & 224 & 270 \\
\hline
\end{tabular}

segundo mês após anel de Ferrara e primeiro, terceiro, e doze meses após "crosslinking", incluindo medida de AV, refração, topografia de córnea com Pentacam (Oculus Pentacam), lâmpada de fenda (Topcon) e microscopia especular da córnea (Microscopia especular de contato Conam)

Todos os procedimentos foram realizados pelo mesmo cirurgião (E.A).

\section{Resultados}

Resultados pós-cirurgia de Anel de Ferrara e pré-crosslinking:

Os pacientes obtiveram melhora de AV com correção, em $100 \%$ dos casos, mas após 3 meses estes casos apresentaram baixa de AV progressiva, como mostra o gráfico 1 e tabela 1 .

O estudo da refração destes pacientes mostrou que 4 olhos (67\%) implantados com anel apresentaram alteração progressiva de refração e 2 olhos (33\%) apresentavam refração sem alteração progressiva, mas haviam sofrido extrusão de anel e reimplante (Gráfico 2).

A topografia (Pentacam) mostra que houve queda acentuada na curvatura máxima destes cones no primeiro mês e não houve progressão significativa nesta curvatura durante um ano em 5 olhos $(83 \%)$, sendo que em um olho (17\%) apresentou alteração 

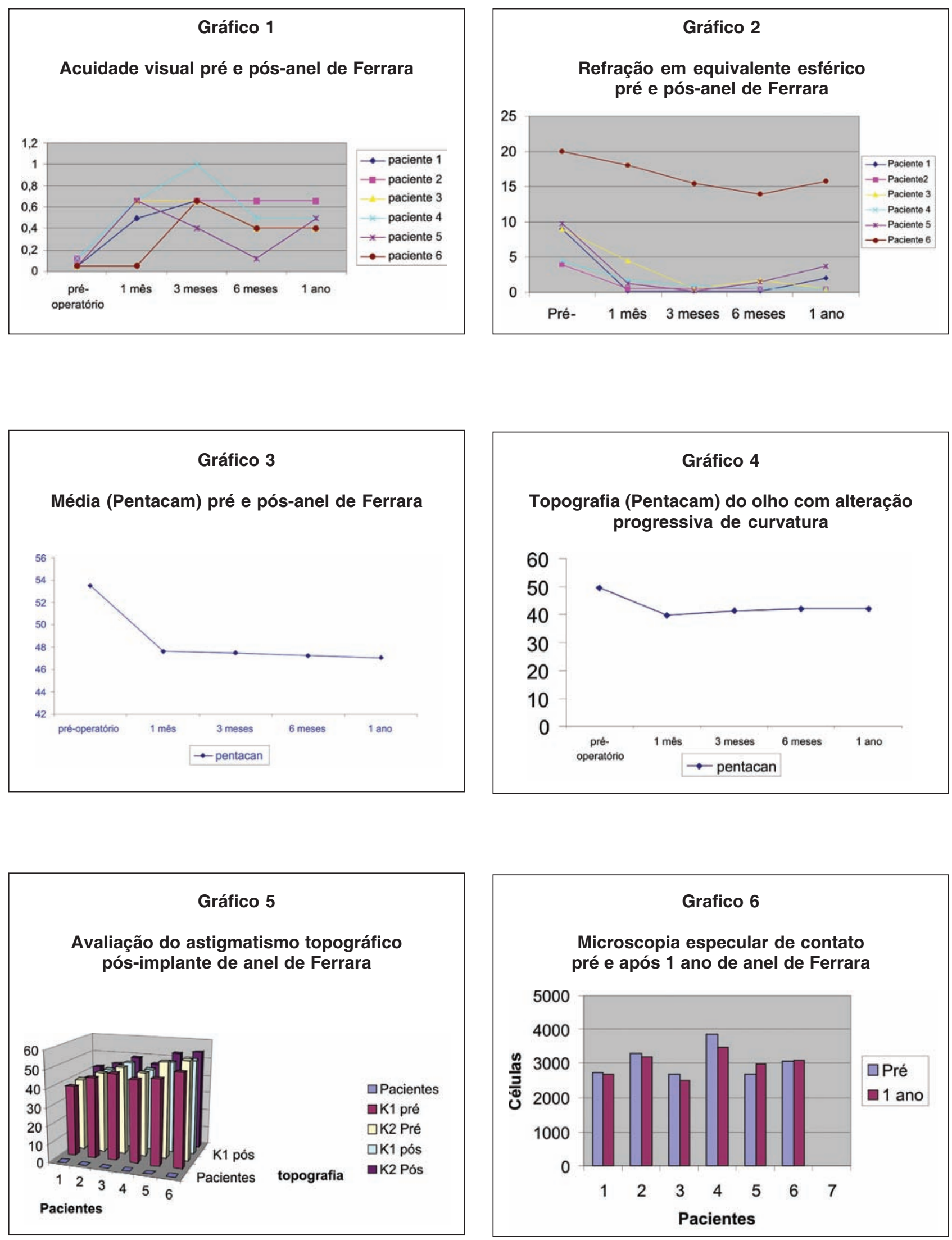

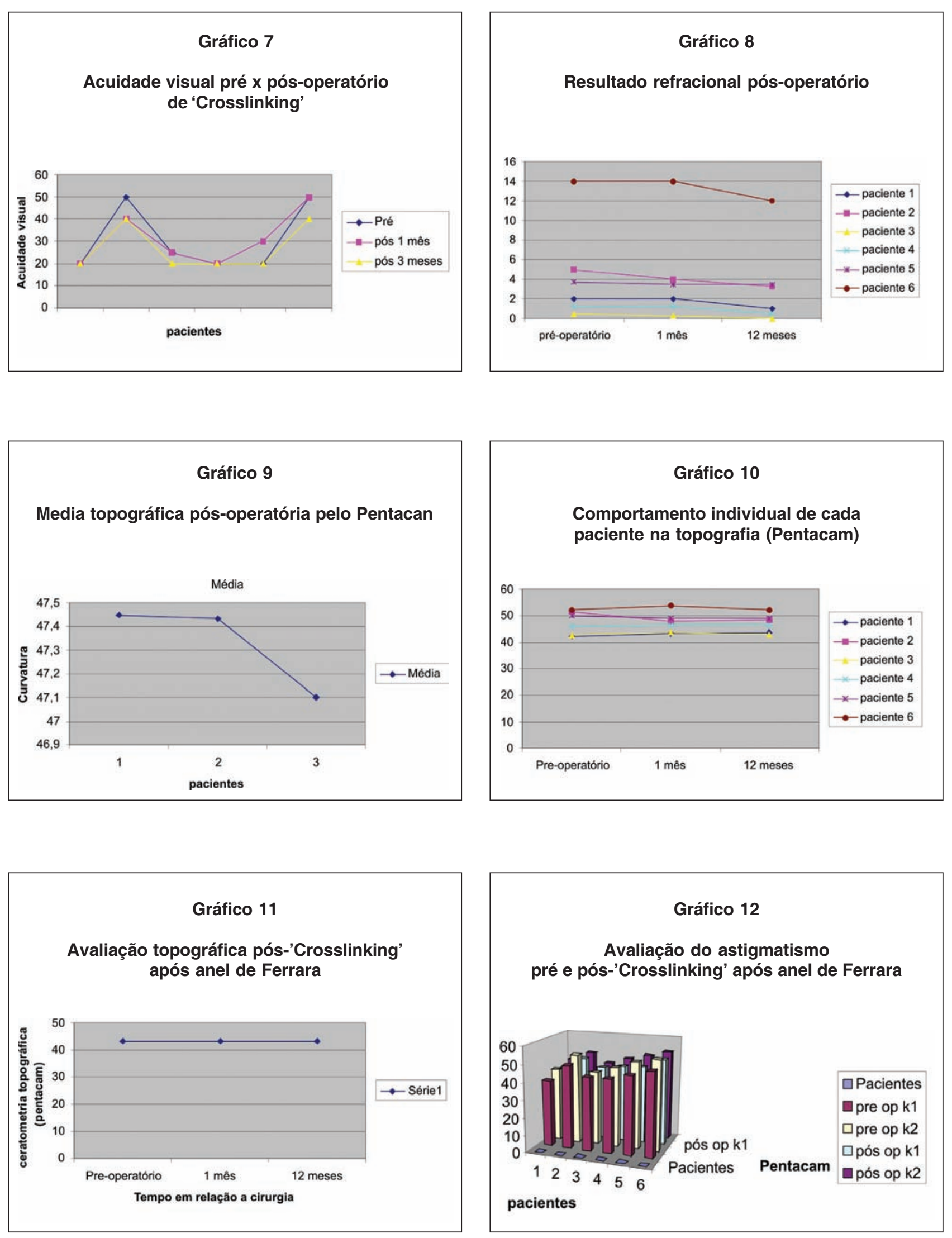


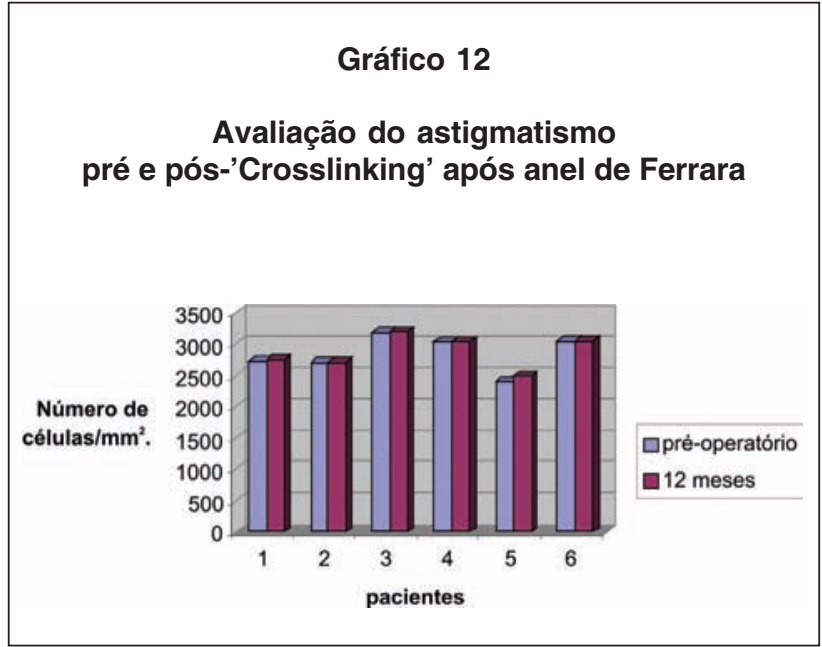

progressiva da curvatura máxima após 3 meses de cirurgia (Gráfico 3 e Tabela 2).

Apenas um olho (17\%) mostra progressão da curvatura máxima (Tabela 3, Gráfico 4).

O astigmatismo topográfico corneano, 12 meses após o implante do anel, mostrou que 5 olhos apresentavam a mesma curvatura máxima pósoperatória de um mês e apenas um olho apresentou aumento da curvatura máxima no pós-operatório tardio. Em 4 pacientes a curvatura máxima se manteve, porém houve aumento na diferença entre o K1 e K2.

O estudo de lâmpada de fenda mostrou anel em boa profundidade durante um ano de pósoperatório em $100 \%$ destes olhos, porém 2 destes olhos tiveram extrusão de um segmento inferior de anel 2,2 e 2,8 anos de pós-operatório de implante de anel intraestromal.

A microscopia especular não mostrava alteração significativa (Gráfico 6).

Resultados pós-crosslinking:

A acuidade visual destes olhos se mostrou semelhante ao pré-operatório tendo apenas ganho máximo de AV de uma linha em 2 casos e ganho de 0,5 linha de visão em um caso.

Em 100\% dos casos não houve perda no equivalente esférico quando comparada a refração pré-operatória com a refração pós-operatória de um ano. Em um caso permaneceu o mesmo equivalente esférico, em 3 casos houve ganho de $0,50 \mathrm{D}$, um caso ganho de $2 \mathrm{D}$ e um caso ganho de $3 \mathrm{D}$ (Gráfico 8 e Tabela 5).

A média topográfica pelo Pentacam mostrou que não houve alteração significativa na curvatura máxima corneana nos pacientes submetidos ao crosslinking. Houve variação de $0,35 \mathrm{D}$ na média destes exames. $\mathrm{O}$ gráfico individual destes pacientes mostrou que a topografia pós-operatória de um ano, permaneceu a mesma do pré-operatório em 4 olhos (66\%), diminuiu 1D em um olho $(17 \%)$ e diminuiu 3 D em um olho (17\%) (Gráfico 9, 10 e Tabela 6).

No caso em que a topografia mostrava evolução progressiva após anel, houve estabilização na curvatura corneana após o crosslinking.

O astigmatismo corneano topográfico revelou que 3 olhos apresentaram a mesma curvatura máxima pré-operatória, um olho diminuiu $2 \mathrm{D}$ na curvatura máxima, um olho diminuiu $1 \mathrm{D}$ na curvatura máxima e apenas um olho apresentou aumento da curvatura máxima de $1 \mathrm{D}$ no pósoperatório tardio, mas os 6 olhos apresentaram menor diferença entre o K1 e K2.

O gráfico mostra diminuição do astigmatismo topográfico em $100 \%$ dos casos.

Lâmpada de fenda:

O estudo de lâmpada de fenda mostrou anel em boa profundidade durante um ano de pósoperatório em $100 \%$ destes olhos, e nenhum destes olhos teve extrusão ou deslocamento de nenhum segmento de anel, com nenhuma modificação de cor ou edema corneano no pós-operatório tardio.

Microscopia Especular:

A microscopia especular também não mostra alteração significativa comparando o pré com o pósoperatório tardio de crosslinking.

\section{DıscussÃo}

Nossa experiência com anel de Ferrara por 11 anos corrobora dados de outros autores, confirmando que o efeito do anel produz queda acentuada da curvatura corneana, trazendo também efeito refrativo, se implantados de acordo com o nomograma de Ferrara ${ }^{(10)}$. O estudo deste grupo comprova o efeito do anel, porém $5 \%$ dos anéis intraestromais implantados, como já se afirma na literatura, mostra algum tipo de intercorrência, como aumento progressivo da curvatura corneana, da refração esférica ou do astigmatismo, extrusão ou deslocamento do anel ${ }^{(5,7)}$. Na nossa casuística em 4 pacientes a curvatura máxima se manteve, porém 
houve aumento na diferença entre o K1 e K2 ocorrendo aumento de astigmatismo corneano, o que justificava a baixa progressiva na acuidade visual e refração em equivalente esférico destes olhos (Gráfico 5). Dos dois pacientes que tiveram extrusão de um segmento do anel, um mostrava aumento progressivo da curvatura corneana e da refração, mesmo após o reimplante (Gráfico 4, Tabela 3). Nestes casos citados, o crosslinking pôde nos auxiliar, mostrando estabilidade da refração em um ano. Não houve nenhuma extrusão, e o tratamento com o crosslinking não propiciou redução significativa da curvatura máxima como ocorre no implante do anel. Nos casos em estudo, verificamos que a maior curvatura corneana após o implante do anel se manteve a mesma curvatura ou houve queda de 1 a $2 \mathrm{D}$ nas ceratometrias máximas após o crosslinking (Gráfico 10, Tabela 6). Porém houve redução significativa no astigmatismo corneano, o que melhorou a refração e a qualidade de visão, mesmo que não tenha trazido melhora acentuada em ganhos de linha de visão (Gráfico 12). A diferença entre K1 e K2 mostrou menor astigmatismo corneano, o que justifica, mesmo sem ganhos de linhas de visão, a melhora que os pacientes referiam na qualidade de visão, comparando o pré com o pós-operatório.

Como o anel mostra melhora refrativa significativa, com melhora da AV e redução acentuada na curvatura corneana e na refração (Gráfico 1-3, Tabela 1-2), e o crosslinking não apresenta a mesma performance refrativa, com alterações mínimas na AV, curvatura corneana e refração (Gráfico 7-10, Tabela 4-6). Não houve nenhuma alteração significativa na microscopia especular destes pacientes, nos mostrando que respeitando as $400 \mu$ de espessura corneana, como relatado na literatura, não há lesão a nível de endotélio $^{(7,11)}$. Nenhuma alteração de cristalino ou retina foram observados nestes casos e nenhuma intercorrência per ou pós-operatória, portanto nenhum efeito adverso, o que nos mostra a segurança da técnica já descrita por vários autores ${ }^{(7,8,11)}$.

\section{Conclusão}

Muito ainda temos que estudar em ceratocone, biomecânica e flexibilidade corneana. Estudos devem ser feitos para colocar os parâmetros de indicação e limites entre as duas técnicas, pois senão estaremos congelando córneas com refrações que podem ser melhoradas com o implante de anel. Faz-se necessário pesquisas para avaliar os limites do crosslinking e do implante do anel. Cremos que isto venha ajudar a normatizar a utilização das duas técnicas. Desta maneira poderemos melhorar a AV naqueles que tenham má qualidade refrativa e depois fazermos o crosslinking para estabilização destas córneas. O crosslinking como primeira escolha deveria ser utilizado apenas nas córneas que tenham boa qualidade refrativa. Mas, devemos sempre abrir um leque para novas tecnologias que vem se despontando.

O crosslinking, técnica de fácil acesso, poderá nos ajudar a manter ceratocones estáveis e cada vez menor as filas de espera para transplantes. Não somente nos cones em progressão, mas também nos cones evolutivos com implante de anel podemos utilizar o crosslinking. Apesar de os resultados serem animadores, consideramos serem iniciais, e devem ser corroborados por outros centros.

\section{Abstract}

Purpose: To describe our experience with crosslinking after Ferrara ring implant for better vision and corneal stabilization. Methods: six patients with age of $26-38$ years old had treatment with crosslinking after Ferrara ring implant. Five patients had 2 ring segments implanted and one patient had one segment implanted. After 4 to 55 months the patients were submitted to crosslinking with UVA light according to Seiler et al protocol. Results: Four eyes had progressive alteration of refraction and 2 patients had no alteration of refraction. The topography showed flattener curvature and at the slip lamp 100\% of the eyes had good depth after one year post operatively. The specular microscopy didn't show any significant alteration and the visual acuity was similar before the procedure. Conclusion: The crosslinking may help to maintain stable keratoconus, not only in progression cases, but also in evolutive keratoconus with ring implant.

Keywords: keratoconus/surgery; Intra corneal ring; Cornea/surgery; Cornea/pathology; Visual acuity; Photosensitizing agents/therapeutic use; Riboflavin/therapeutic use; Ultraviolet rays; Corneal topography 


\section{REFERÊNCIAS}

1. Kwitko S, Severo NS. Ferrara intracorneal ring segments for keratoconus. J Cataract Refract Surg. 2004;30(4):812-20.

2. Lovisolo CF, Fleming JF. Intracorneal ring segments for iatrogenic keratectasia after laser in situ keratomileusis or photorefractive keratectomy. J Refract Surg. 2002;18(5):535-41.

3. Kanellopoulos AJ, Pe LH, Perry HD, Donnenfeld ED. Modified intracorneal ring segment implantations (INTACS) for the management of moderate to advanced keratoconus: efficacy and complications. Cornea. 2006;25(1):29-33.

4. Alio JL, Shabayek MH, Artola A. Intracorneal ring segments for keratoconus correction: long-term follow-up. J Cataract Refract Surg. 2006;32(6):978-85.

5. Kymionis GD, Siganos CS, Tsiklis NS, Anastasakis A, Yoo SH, Pallikaris AI, et al. Long-term follow-up of Intacs in keratoconus. Am J Ophthalmol. 2007;143(2):236-44.

6. Wollensak G, Spoerl E, Seiler T. Riboflavin/ultraviolet-a-induced collagen crosslinking for the treatment of keratoconus. Am J Ophthalmol. 2003;135(5):620-7.

7. Spoerl E, Mrochen M, Sliney D, Trokel S, Seiler T. Safety of UVA-riboflavin cross-linking of the cornea. Cornea. 2007;26(4):385-9.
8. Hafezi F, Kanellopoulos J, Wiltfang R, Seiler T. Corneal collagen crosslinking with riboflavin and ultraviolet A to treat induced keratectasia after laser in situ keratomileusis. J Cataract Refract Surg. 2007;33(12):2035-40. Comment in: J Cataract Refract Surg. 2008;34(6):879; author reply 879 .

9. Spoerl E, Wollensak G, Seiler T. Increased resistance of crosslinked cornea against enzymatic digestion. Curr Eye Res. 2004;29(1):35-40.

10. Miranda D, Sartori M, Francesconi C, Allemann N, Ferrara P, Campos M. Ferrara intrastromal corneal ring segments for severe keratoconus. J Refract Surg. 2003;19(6):645-53.

11. Seiler T, Hafezi F. Corneal cross-linking-induced stromal demarcation line. Cornea. 2006;25(9):1057-9.

\author{
ENDEREÇO PARA CORRESPONDÊNCIA: \\ Edna Almodin \\ Rua Silva Jardim, no 359 - Centro \\ CEP 87013-000 - Maringá -PR - Brasil \\ TEL: 44 3262-2061 FAX:44 3225-1162
}

